\title{
Regulierung und Deregulierung des „kleinen Unterschieds": Nachtarbeitsverbot und Konstruktion von Geschlecht, 1864-1930
}

\author{
Regina Wecker
}

I.

Die Delegierten des Internationalen Arbeiterschutzkongresses, der 1897 in Zürich stattfand, waren sich über die wesentlichen Forderungen zur Verbesserung der Arbeitsbedingungen in den Fabriken einig. Dazu gehörte der gesetzliche Maximalarbeitstag - längerfristig sollte der Achtstundentag zur Regel werden --, ein arbeitsfreier Sonntag, die Festsetzung eines Mindestalters für Kinder, Unfallschutz und Schutzmaßnahmen bei der Handhabung von giftigen Stoffen. ${ }^{1}$ Die 300 Delegierten der Gewerkschaften beschlossen diese Maßnahmen einstimmig. Neben den Gewerkschaftsdelegierten nahmen am Kongress etwa 100 Gäste teil, Abgesandte der Regierungen und bekannte Sozialpolitiker - die "geistige Elite" Europas war in Zürich versammelt, wie es im Urteil der Zeitgenossen hieß. Die prinzipielle Übereinstimmung über die Notwendigkeit der Maßnahmen an den ersten drei Kongresstagen war so auffallend, dass der deutsche Sozialdemokrat Wilhelm Liebknecht von einem „Gottesfrieden" sprach.

Dann aber "entfesselte" am vierten Verhandlungstag der belgische Delegierte De Wiart, Wortführer der Demokratisch-christlich-socialen Arbeiterpartei, einen ,Sturm“2 - wie es der Berichterstatter formulierte:

Der Prinzipienwind von gestern ist heute zum Sturm angewachsen. Eine parlamentarische Redeschlacht in des Wortes vollster Bedeutung ward geschlagen zwischen beiden Heerlagern des Kongresses; die besten Sprecher auf beiden Seiten traten in die Schranken und die Leidenschaften im Saal wie auf den Tribünen wurden aufs Höchste erregt. ${ }^{3}$

DeWiart, der diesen Sturm der Leidenschaften ausgelöst hatte, forderte: „Die Arbeit der Frauen, namentlich die Arbeit verheirateter Frauen in den Bergwerken, Steinbrüchen und in der Großindustrie soll allmählich

1 Vgl. Gustav Meier, Der internationale Kongress für Arbeiterschutz in Zürich vom 23.-28. August 1897, Bern 1897, 21.

2 Meier, Kongress, wie Anm. 1, 22.

3 Meier, Kongress, wie Anm. 1, 21. 
abgeschafft werden." ${ }^{4}$ Er begründete den Antrag ,in ausserordentlich geschickter Weise", wie der Berichterstatter festhält:

Die durch den Kapitalismus herbeigeführte Frauenarbeit ist als Ungeheuerlichkeit, als eine Verleugnung des historischen Fortschritts, als eine Versündigung gegen die Natur zu bezeichnen. Die physische Konstitution der Frau ist von unbestreitbarer Schwäche; die Fabrikarbeit ist mit derselben ganz unvereinbar. ... Das Argument, dass die Frauenarbeit zur Erhaltung der Familie nötig sei, erinnere inn immer an Dante's Grafen Ugolino im Hungerturm, der seine mit inm eingesperrten Kinder auffresse, um innen den Vater zu erhalten. Die Frauenarbeit ist eine der Hauptursachen des Zerfalls der menschlichen Gesellschaft. Sie ist, wie auch die Kinderarbeit erst zu Anfang dieses Jahrhunderts unter dem Regime von Robert Peel in England eingeführt worden, um die Löhne der Männer zu drücken. ${ }^{5}$

Nach einer langen Diskussion, in der sich auch Lily Braun und Clara Zetkin ,entschieden" gegen den Antrag ausgesprochen hatten, wurde angesichts einer immer länger werdenden Rednerliste beschlossen, die Kontroverse um den Ausschluss der Frauen nur noch von je einem Redner vertreten zu lassen. Als Redner gegen den Ausschluss wurde der deutsche Sozialdemokrat August Bebel nominiert, für den Ausschluss von Frauen votierte der Schweizer Caspar Decurtins, katholischkonservativer Nationalrat aus Graubünden. Bebels Haltung, dass Fabrikarbeit der Frauen für die Arbeiterfamilien eine ökonomische Notwendigkeit darstellte, dass es aber wichtig war, Frauen noch stärker als Männer durch gesetzliche Regelungen vor Gesundheitsschädigung und Ausbeutung zu schützen, überzeugte die Delegierten. Der Antrag de Wiart wurde mit 165 : 98 abgelehnt. Ebenso deutlich abgelehnt wurde auch ein Antrag der Französin Marie Bonieval, Delegierte der Pariser Lehrerinnen-Gewerkschaft. Sie hatte verlangt, auf einen Sonderschutz für Frauen zu verzichten und Schutzgesetze nur soweit zuzulassen, wie sie für beide Geschlechter gelten.

Dieser Streit ist einer der Höhepunkte, aber auch wohl der Schlusspunkt der Auseinandersetzung um die gesetzliche Abschaffung der Arbeit von verheirateten Frauen in Fabriken. Drei Möglichkeiten wurden hier nochmals auf der internationalen Bühne diskutiert: der Ausschluss vor allem der verheirateten Frauen, die spezielle Reglementierung der weiblichen Arbeitskraft durch Schutzgesetze und die Gleichstellung von Männern und Frauen. Die Entscheidung für den Sonderschutz kann also als Kompromissvorschlag zwischen den beiden ,Extremen' gesehen werden, als Ausweg gegenüber der Forderung der Abschaffung und der völligen Gleichbehandlung. Im Folgenden werde ich die Entstehung des Frauenschutzes nachzeichnen und inn sowohl in seiner diskursiven als auch in seiner strukturellen Bedeutung für die Geschlechterordnung analysieren. Mein besonderes Interesse gilt den Arbeitszeitregelungen und dem Nachtarbeitsverbot in Deutschland und in der Schweiz.

4 Internationaler Kongress für Arbeiterschutz, Zürich 1897, Amtlicher Bericht, Zürich 1898, 206.

5 Meier, Kongress, wie Anm. 1, 22. 
II.

Die nationalen Diskussionen, Maßnahmen und Gesetze gingen der Debatte auf der internationalen Bühne voraus. In einer ersten Phase waren Fabrikgesetze Kinderschutzgesetze: 1815 wurde in Zürich damit begonnen, die Fabrikarbeit für Kinder einzuschränken. Es folgten weitere Schweizer Kantone. 1819 und 1833 schuf England gesetzliche Grundlagen, 1837 Preußen. Im Wesentlichen wurde in diesen Gesetzen die Arbeitszeit für Kinder auf etwa zehn bis zwölf Stunden festgelegt, Nachtarbeit verboten oder eingeschränkt und es wurde ein Minimalalter bestimmt, meist neun oder zehn Jahre.

Noch größere Widerstände als bei den Kinderschutzgesetzen, die aus ökonomischen Gründen auch von den Eltern bekämpft wurden, gab es bei den ersten Versuchen der gesetzlichen Regelung der Arbeitszeit der Männer. Dagegen wurde nicht nur mit ökonomischen Erwägungen, sondern auch mit dem Prinzip der Vertragsfreiheit argumentiert. Zentral war dabei der Grundsatz, dass die Vertragspartner als autonome individuen, als free agents, die Vertragsbedingungen ohne gesetzliche Begrenzung aushandeln sollten. Dieses Prinzip tangierte auch die politischen Rechte. Besonders in England, wo das Wahlrecht durch Census-Bestimmungen eingeschränkt war, hätte die Abhängigkeit des besitzlosen Fabrikarbeiters als Argument gegen die Ausweitung der politischen Rechte gebraucht werden können. Es musste verhindert werden, dass die Fabrikgesetzgebung die Vorstellungen von Abhängigkeit - und damit politischer Unmündigkeit - stärkte. ${ }^{6}$

Gegenüber Frauen, die nicht als unabhängige Vertrags- und Verhandlungspartner im juristisch-politischen Sinn galten, schienen diese Rücksichten nicht nötig: 1844 wurden in England erstmals die Arbeitsbedingungen in Fabriken für Frauen geregelt, 1847 wurde der Zehnstundentag beschlossen und 1853 das Nachtarbeitsverbot für Frauen und Kinder. ${ }^{7}$ Man kann das durchaus als doppelte Strategie werten: Einerseits wurde damit ein deutlicher Unterschied zwischen der, Verwertbarkeit' und damit dem Wert der weiblichen und der männlichen Arbeitskraft geschaffen, andererseits hoffte man durch die Reduktion der Arbeitszeit der Frauen dem allgemeinen Zehnstundentag näher zu kommen, ohne dass die staatsbürgerlichen ,Qualitäten' der Männer in Frage gestellt werden mussten, da unterschiedliche Arbeitszeiten von Männern und Frauen die Arbeitsabläufe behinderten. ${ }^{8}$

In der Schweiz konnte man die Etablierung eines Normalarbeitstags direkter angehen. 1864 wurde in der Landsgemeinde des Kantons Glarus, der Versammlung aller stimmberechtigten Männer, ein Fabrikge-

6 Erst der Reform Act von 1867 setzte die Besitzqualifikationen so fest, dass sie ein großer Teil der städtischen Arbeiterschaft erfüllen konnte.

7 Für den Wortlaut der Gesetze vgl. Ursula R. Henriques, Before the Welfare State, London 1979, Kap. 4-5.

$8 \mathrm{Vgl}$. Jane Lewis u. Sonya O. Rose, "Let England Blush": Protective Labour Legislation, 1820-1914, in: Jane Lewis u. a. Hg., Protecting Women. Labor Legislation in Europe, the United States, and Australia, 1880-1920, Urbana/Chicago 1995, 95124, hier $95 \mathrm{ff}$ und Robert Gray, Languages of Factory Reform, in: Patrick Joyce Hg., The Historical Meanings of Work, Cambridge 1987, 143-179, 64. 
setz beschlossen, das - außer Kinderschutzbestimmungen - einen Zwölfstundentag, ein Nachtarbeits- und Sonntagsarbeitsverbot enthielt. Damit war der erste Maximalarbeitstag im Sinne einer für beide Geschlechter geltenden Regelung geschaffen. Das Gesetz ging auf eine Eingabe von Fabrikarbeitern zurück. Sie hatten einen allgemeinen Elfstundentag und eine Fabrikinspektion gefordert. Allerdings war im vorberatenden Parlament versucht worden, die Forderung auf die Einführung eines Zwölfstundentags für Frauen und eines Nachtarbeitsverbots für Frauen und Kinder zu reduzieren. ${ }^{9}$ Argumentiert wurde hier ebenfalls mit der Vertragsfreiheit. Dieser Versuch war wohl nicht zuletzt darum erfolglos, weil das allgemeine Männerwahlrecht in Glarus seit 1836 und in der Schweizer Bundesverfassung seit 1848 fest verankert und der Konnex Vertragsfreiheit und politische Rechte nicht mehr herstellbar war. Die in der Landsgemeinde, der Versammlung aller Wahlberechtigten, direkt vertretenen Arbeiter legten - unterstützt von Pfarrern und Arzten - die staatliche Regulierung, den Zwölfstundentag und ein Nachtarbeitsverbot für alle Arbeitskräfte fest. ${ }^{10}$ Damit schufen sie eine Schweizer Tradition, die wohl einzigartig im internationalen Vergleich ist: ein Nachtarbeitsverbot für Männer. Neben der Tatsache, dass das allgemeine Wahlrecht nicht zur Disposition stand, spielte dabei die direkte Beteiligung der Fabrikarbeiter im Prozess der Gesetzgebung sowie die spezielle Atmosphäre einer Landsgemeinde und damit ein gewisser Gruppendruck eine Rolle. ${ }^{11}$ Ebenso wichtig scheint der hohe Frauenanteil in der Glarner Fabrikindustrie gewesen zu sein. In Glarus waren in der Mitte des 19. Jahrhunderts weit mehr als die Hälfte der erwachsenen Belegschaft der Fabriken Frauen. Jedes Fabrikgesetz galt also mehrheitlich für Frauen. Dass die stimmberechtigten Männer - in der Fabrikindustrie die Minderheit - für ihren eigenen Schutz durch Gleichstellung mit den Frauen sorgten, bedeutet auch, dass sie offensichtlich, anders als ihre englischen Kollegen, zu dieser Zeit kein Interesse am Ausschluss von Frauen aus der Industrie hatten. Man war auf das Einkommen der Frauen dringend angewiesen. Fabrikarbeit war weniger stark männlich konnotiert, und Schutz nicht ein ausschließlich weibliches Bedürfnis. Nur ein Gesetzesparagraf betraf allein Frauen: das Verbot, während sechs Wochen nach der Niederkunft in den Fabriken zu arbeiten. ${ }^{12}$

9 Vgl. Heinz Dällenbach, Kantone, Bund und Fabrikgesetzgebung, Zürich 1961, 72ff. Zur Entstehung und zur Urheberschaft der Eingabe der Glarner Arbeiterschaft vgl. auch Peter Stolz, Politische Entscheidungen in der Versammlungsdemokratie, Bern $1968,108 \mathrm{ff}$

$10 \mathrm{Vgl}$. Regina Wecker, Equality for Men? Factory Laws, Protective Labor Legisiation for Women in Switzerland, and the Swiss Effort for International Protection, in: Lewis, Women, wie Anm. 8, 63-90, hier 68ff. Zur Geschichte der Schutzgesetzgebung in der Schweiz im 20. Jahrhundert vgl. Regina Wecker, Brigitte Studer u. Gabi Sutter, "Die schutzbedürttige Frau". Zur Konstruktion von Geschlecht durch Mutterschaftsversicherung, Nachtarbeitsverbot und Sonderschutzgesetzgebung, Zürich (erscheint demnächst).

11 Vgl. Stolz, Entscheidungen, wie Anm. 9, $112 f f$

12 Vgl. Gesetz über die Fabrikpolizei, 1864, Amtliche Sammlung der Gesetze und Verordnungen des Kantons Glarus, Glarus 1864. 
Die Schweiz blieb für die spätere Gesetzgebung zunächst bei diesem allgemeinen, beide Geschlechter betreffenden Schutz. Die erste nationale Gesetzgebung, das Eidgenössische Fabrikgesetz von 1877 , verankerte als Normalarbeitstag den Elfstundentag und das allgemeine Nachtarbeitsverbot für beide Geschlechter. Aber es wurden flexible Ausnahmen für männliche Arbeitskräfte eingebaut: „Bei Fabrikationszweigen, die ihrer Natur nach einen ununterbrochenen Betrieb erfordern, kann regelmässig Nachtarbeit stattfinden."13 Dies galt ausschließlich für Männer. Frauen sollten „unter keinen Umständen“ zur Sonntags- oder Nachtarbeit "verwendet werden". ${ }^{14}$

Als Neuerung gegenüber den kantonalen egalitären Anfängen wurde 1877 ins Eidgenössische Fabrikgesetz eine Bestimmung über Fabrikationszweige aufgenommen, in denen schwangere Frauen nicht arbeiten dürfen. Auch wurde allen Frauen verboten, „im Gange befindliche Motoren" und „gefahrdrohende Maschinen" zu reinigen. ${ }^{15}$ Weitere Sonderbestimmungen waren eine verlängerte Mittagspause für diejenigen, die „ein Hauswesen zu besorgen hatten", ${ }^{16}$ aber auch die Bestimmung, dass verheiratete Frauen keine Hilfsarbeiten verrichten durtten, die über die elfstündige Arbeitszeit hinausgingen. ${ }^{17}$ Das Arbeitsverbot während des Wochenbettes wurde auf acht Wochen festgesetzt, davon sechs Wochen nach der Geburt.

Damit war der „kleine Unterschied“, die Charakteristiken der Frau als ,besondere' Arbeitskraft gesetzlich - wenn auch noch vorsichtig - vorgezeichnet. Mit diesen Sonderbestimmungen wurde ein weibliches Wesen konstruiert, das weniger ausdauernd war und zudem ungeeignet, mit Maschinen umzugehen. Damit mangelten ihr wesentliche Merkmale, die in der neuen industrialisierten Welt entscheidend wurden. Das „Hauswesen" wurde gegenüber der Fabrik für Frauen zur vorrangigen Wirkungsstätte deklariert. Es bahnt sich hier ein Prozess des diskursiven gendering der Fabrikarbeit als männlicher Arbeit an, als dessen logische Folge ein Antrag auf Ausschluss, wie der De Wiarts, gesehen werden kann. Interessant für den Schweizer Kontext ist dabei, dass sich dieses gendering auf der quantitativen Ebene schon länger abzeichnete: Während die Mehrzahl der Fabrikarbeiterschaft in der Anfangsphase der Industrialisierung weiblich war, nahm der Anteil der Frauen gegen Ende des 19. Jahrhunderts deutlich ab. Gleichzeitig wurden Frauen als Geschlechtswesen normiert. Zunächst wird ihnen während der Schwangerschaft der Umgang mit bestimmten Giften verboten, in der späteren Gesetzgebung wird dieses Verbot auf alle Frauen ausgedehnt. Argumentiert wird mit dem Schutz des ungeborenen Kindes und mit der reproduktiven Aufgabe der Frauen, aller Frauen. Die Notwendigkeit, männliche Arbeiter vor dem Umgang mit Giftstoffen zu schützen, ist hingegen nie damit begründet worden, dass Gifte die Sexualität und die Zeugungsfähigkeit von Männern

13 Bundesgesetz betreffend die Arbeit in den Fabriken vom 23.3.1877, Art. 13. Amtliche Sammlung, III, 1879 (Fabrikgesetz 1877).

14 Fabrikgesetz 1877, Art. 15, wie Anm. 13.

15 Fabrikgesetz 1877, Art. 15, wie Anm. 13.

16 Fabrikgesetz 1877, Art. 15, wie Anm. 13.

17 Vgl. Fabrikgesetz 1877, Art. 11, wie Anm. 13. 
beeinträchtigen. Männer werden nicht in ihrer Sexualität und als Geschlechtswesen geschützt, sondern als Arbeitskräfte.

In Deutschland hatte man zwar das Schweizer Fabrikgesetz zum Vorbild genommen, den Normalarbeitstag aber nicht durchgesetzt. ${ }^{18}$ Auch die deutsche Schutzgesetzgebung, die in die Gewerbeordnungsnovelle von 1891 mündete, hatte ursprünglich eine umfassende Ordnung der Fabrikverhältnisse zum Ziel. ${ }^{19}$ Die Novelle blieb jedoch weit hinter der Diskussion zurück. Die allgemeinen Bestimmungen zur Begrenzung der Arbeitszeit waren sehr schwammig, eher Empfehlungen als bindende Regelungen. Die von der SPD geforderte Festlegung der Maximalarbeitsdauer war nicht angenommen worden. „Durch Beschluss des Bundesrates" sollte für die Gewerbe, ,in welchen durch eine übermäßige Dauer der täglichen Arbeitszeit die Gesundheit der Arbeiter gefährdet wird, die Dauer der zulässigen täglichen Arbeitszeit und der zu gewährenden Pausen vorgeschrieben werden. "20 Allerdings wurden Arbeitervertretungen in den Fabriken geschaffen.

Die Frauenartikel der Novelle aber legten den Elfstundentag für Frauen fest und enthielten zudem die Elemente, die ich für die 14 Jahre vorher verabschiedete nationale Schweizer Gesetzgebung erwähnt habe: Das 1878 eingeführte Beschäftigungsverbot für Wöchnerinnen wurde von drei auf vier Wochen erhöht, neu waren das Nachtarbeitsverbot, die verlängerte Mittagspause „auf Antrag“. Damit waren die Kernbereiche der Novelle Bestimmungen, die ausschließlich die Frauenarbeit reglementierten. 1908 wurde die Arbeitszeit der Frauen in Deutschland auf zehn Stunden reduziert.

Der Novelle kommt eine zentrale Rolle in der deutschen Sozialpolitik zu. ${ }^{21}$ Sie markiert eine deutliche Ausweitung der staatlichen Sozial- und Familienpolitik. Trotz der Beschränkung der Arbeitszeitregelungen auf weibliche Arbeitskräfte beanspruchte sie, sich auf die "ganze Nation" zu beziehen. Die Gesundheit der Nation sollte stellvertretend durch den Schutz der Arbeiterinnen verbessert werden. Tatsächlich strukturierte die Novelle die Arbeitswelt neu. Sie legte Frauen auf ihre Rolle als Objekte der Gesetzgebung fest, während Männer in ihrer Rolle als handelnde Subjekte und Vertragspartner gestärkt wurden: Ihnen wurde „zugestanden", selbst für ihre Rechte als Arbeitskräfte einzustehen, zudem wurden ihre politischen Rechte innerhalb der Fabrik erweitert. Kurzfristig und individuell mag die Beschränkung des Schutzes auf Frauen für Männer nachteilig gewesen sein und der Ausbeutung ihrer Arbeitskraft Vorschub geleistet haben. Längerfristig aber stärkte die Regelung die Position des Mannes als Vertragspartner auf dem Arbeitsmarkt und als Staatsbürger.

18 Vgl. Sabine Schmitt, „All these Forms of Women's Works which Endanger Public Health and Public Welfare". Protective Labor Legislation for Women in Germany, 1878-1914, in: Lewis, Women, wie Anm. 8, 125-149, hier 127.

19 Vgl. Schmitt, Forms, wie Anm. 18, 127ff, sowie Hans-Jörg von Berlepsch, „Neuer Kurs" im Kaiserreich? Die Arbeiterpolitik des Freiherrn von Berlepsch 1890-1896, Bonn 1987, 146ff.

20 Berlepsch, Kurs, wie Anm. 19, 197.

21 Vgl. dazu Kathleen Canning, Languages of Labour and Gender. Female Factory Work in Germany, 1850-1914, New York 1996, Kap. 4. 
Die Schweizer Gesetzgebung, die die Arbeitszeit für Männer und Frauen gleichermaßen regelte, behielt weiterhin Ausnahmecharakter und schien dadurch gefährdet. Der Normalarbeitstag wurde, wie es der hoch angesehene ehemalige Schweizer Fabrikinspektor Schuler 1897 ausdrückte, „zum schwersten Stein des Anstosses", „,zum Unikum", das die Konkurrenzfähigkeit der Schweizer Industrie zu gefährden schien, und man hätte inn darum wohl am liebsten - zumindest als eine auch Männer betreffende Regelung - wieder aufgehoben. Die Schutzgesetzgebung für Frauen wurde dagegen kaum angegriffen. Schuler erläuterte dazu in seiner Schrift Das Fabrikgesetz und die Konkurrenzfähigkeit der Schweizerischen Industrie:

Man verweist auf die meisten andern Länder, wo die Männer frei arbeiten dürfen. Selten wagt sich Einer mit dem Wunsch an die Öffentlichkeit, dass auch für Frauen und Kinder wieder jede Schranke falle, denn die Notwendigkeit dieses Schutzes wagt doch niemand mehr zu leugnen, nachdem die ganze zivilisierte Welt sie durch ihre Gesetzgebung anerkannt, nachdem die meisten Gesetze sogar noch engere Schranken gegenüber jedem Übermass gezogen. ${ }^{22}$

Die Schutzgesetze für Frauen wurden also auch in der Schweiz von den Gegnern staatlicher Regelungen, die durch die deutsche Gesetzgebung wieder Auftrieb erhalten hatten, kaum in Frage gestellt. Der Arbeitsmarkt war zunehmend zum Ort avanciert, an dem ökonomische Chancen und gesellschaftliche Dominanz festgelegt wurden. Folgerichtig musste die Geschlechterordnung auch in der wirtschaftlichen Ordnung sichtbar gemacht, die Geschlechterdifferenz auf dem Arbeitsmarkt akzentuiert werden. Schutzgesetze wurden zu einem Mittel, diese Differenz zu betonen.

Am Nachtarbeitsverbot und seinen Folgen lässt sich sowohl die strukturelle als auch die diskursive Wirkung dieser Schutzbestimmungen besonders gut erkennen. In Deutschland war Nachtarbeit auch schon vor der Einführung der Gewerbenovelle für Frauen eher unüblich. ${ }^{23}$ Die Durchführung und Kontrolle des Verbots war daher meist unproblematisch. Eine Ausnahme bildete die Zückerindustrie. Auf Grund des Saisonbetriebs und der Notwendigkeit der schnellen Verarbeitung der Zuckerrüben war Nachtarbeit von Frauen hier üblich. Man beschäftigte mehrheitlich Wanderarbeiterinnen. Die Zuckerrübenindustrie war dann auch die einzige Industrie, die gesamthaft eine Ausnahmebewilligung erhielt, allerdings wurde diese Bewilligung an die Auflage der sukzessiven Abschaffung der Nachtarbeit und an zusätzliche Maßnahmen gebunden, wie z. B. ein Verbot, dass Frauen die technischen Einrichtungen bedienten. Interessant ist nun - wie Sabine Schmitt nachgewiesen hat ${ }^{24}$-, dass die Industrie die Ausnahmebewilligung bei

22 Fridolin Schuler, Das Fabrikgesetz und die Konkurrenzfähigkeit der Schweizerischen Industrie, 1897, in: Ausgewählte Schriften von Fabrikinspektor Dr. Fridolin Schuler, hg. von Heinrich Wegmann, Karlsruhe 1905, 92. Zur Haltung Schulers vgl. Regina Wecker, Normalarbeitstag, Gesundheitsschutz und Nachtarbeitsverbot: Zwischen Schutz und Konstruktion von Weiblichkeit und Männlichkeit, in: Hansjörg Siegenthaler $\mathrm{Hg}$., Wissenschaft und Wohlfahrt, Zürich 1997, $217-238$.

$23 \mathrm{Vgl}$. Schmitt, Forms, wie Anm. 18, 159ff

$24 \mathrm{Vgl}$. Schmitt, Forms, wie Anm. 18, 162. 
weitem nicht ausnützte, sondern den Arbeitsablauf restrukturierte, Frauen entließ und Männer einstellte und somit die Nachtarbeit viel schneller als gefordert einschränkte. Die weniger flexible Arbeitskraft der Frau wurde ersetzt, und damit der Prozess des gendering deutlich beschleunigt. Dass die Entlassungen, die von der Industrie mit den unzumutbaren gesetzlichen Auflagen begründet wurden, nicht größeres Aufsehen erregten, lag wohl daran, dass sie vielfach in- und ausländische Wanderarbeiterinnen betrafen.

Im Ersten Weltkrieg setzten sowohl die Krieg führenden Länder als auch die Schweiz die Schutzbestimmungen außer Kraft. ${ }^{25}$ Damit wurde auch Nachtarbeit für Frauen wieder zulässig. Nach Kriegsende erlangten die Schutzbestimmungen wieder Gesetzeskraft. In Deutschland wurde Ende 1918 nun erstmals auch die Arbeitszeit von Männern gesetzlich festgelegt, der Achtstundentag wurde zum Normalarbeitstag und zwar für alle gewerblichen und öffentlichen Betriebe einschließlich des Bergbaus. ${ }^{26}$ Der Normalarbeitstag war in Deutschland nun zwar gesetzlich festgelegt, wurde aber bald in Frage gestellt und durch die Arbeitszeitverordnung vom 21.12.1923, durchlöchert'. ${ }^{27}$ Sie erlaubte die ,freiwillige' Ausdehnung der Höchstarbeitszeit bis auf zehn Stunden, bereitete den Boden für eine viel ausgedehntere Arbeitszeit vor, die nicht selten bis in die Nacht ging.

In der Schweiz war die Sache etwas komplizierter. Das Fabrikgesetz betraf nur die Fabrikindustrie im engeren Sinn; hier wurde nach dem Krieg der Zehnstundentag eingeführt. Die Schaffung eines Gewerbegesetzes, das auch die Arbeitsverhältnisse von Männern außerhalb der Fabriken reglementiert hätte, war um die Jahrhundertwende eines der Ziele der Sozialpolitik. Fabrikinspektor Schuler begründete die Notwendigkeit eines umfassenden Schutzes wie folgt:

Was die Männer anbetrifft, darf wohl zuerst gefragt werden: sind sie denn immer frei, wenn sie zu übermäßiger Überzeit ,ja" sagen? Und weiter: ist es wohl ein Vorteil für unsere Industrie, wenn man den Männern zu viel zumutet? ... Die Antwort wird wohl heute lauten wie vor zwanzig Jahren: wir schützen die Männer im Interesse der Arbeiterschaft, im Interesse der Industrie!28

Die Schaffung eines umfassenden Gesetzes gelang in der Schweiz tatsächlich erst 1964. Das Fehlen von gesetzlichen Regelungen schien einigen Kantonen aber in Bezug auf die weiblichen Arbeitskräfte unzumutbar. Sie schufen so genannte Arbeiterinnenschutzgesetze, Gesetze, die die Arbeitsbedingungen von Frauen außerhalb des Fabriksektors regelten. Basel-Stadt hatte als erster Kanton 1884 ein Gesetz eingeführt, das ähnlich dem Fabrikgesetz, die Arbeitszeit für Frauen auf elf Stunden begrenzte und die Nacht- und Sonntagsarbeit untersagte. ${ }^{29}$ Als Begrün-

25 In Deutschland mit dem „Gesetz betreffend Ausnahmen von Beschäftigungsbeschränkungen“ vom 4. August 1914, in: Reichsgesetzblatt 1914, 333-334.

26 Vgl. Demobilmachungsordnung vom 23.11.1918, Ergänzung vom 17.12.1918.

27 Vgl. Stefan Bajohr, Die Hälfte der Fabrik. Geschichte der Frauenarbeit in Deutschland 1914-1945, Marburg 1979, 192.

28 Schuler, Fabrikgesetz, wie Anm. 22, 92.

$29 \mathrm{Vgl}$. Gesetz betreffend die Arbeitszeit der weiblichen Arbeiter 1884 und Gesetz betreffend den Schutz der Arbeiterinnen vom 23.4.1888, Amtliche Sammlung BaselStadt. 
dung wurde angeführt, dass es nicht einzusehen war, warum die Arbeitszeit in Fabriken auf elf Stunden begrenzt wurde, aber in Ateliers und Werkstätten, wo z. T. schlechtere Arbeitsbedingungen herrschten, keine Maximalarbeitszeit festgesetzt war. Diese Argumentation wäre wohl auch für die Arbeitssituation von Männern zutreffend gewesen und hätte ein allgemeines Gewerbegesetz legitimiert. Es wurde aber nur auf Frauen bezogen. Dabei blieben weiterhin wichtige Arbeitsbereiche ausgespart: z. B. der Verkauf, das Gaststättengewerbe und ,selbstverständlich' die privaten Haushalte, obwohl dort anerkanntermaßen die längsten Arbeitszeiten üblich waren.

III.

Solchen Tendenzen entgegenzuwirken und damit umfassend für die Schaffung des sozialen Friedens zu wirken, war das Ziel internationaler Regelwerke, wie sie nach dem Ersten Weltkrieg geschaffen wurden. Die Arbeitskonferenz von Washington im Jahr 1919 setzte im Prinzip die Bestrebungen der internationalen Arbeiterschutzkonferenzen fort und schuf verbindliche Verträge und Konventionen, zu deren Einhaltung Staaten, die ihnen beitraten, verpflichtet waren. Die International Labour Organisation (ILO) in Genf ist die Folgeorganisation der nach dem Ersten Weltkrieg geschaffenen internationalen Behörde; sie garantiert bis heute die Einhaltung dieser Konventionen und Verträge. Es wurde in jeweils getrennten Konventionen der Achtstundentag, die Arbeitslosenversicherung, die Sicherung des Einkommens während Schwangerschaft und Wochenbett, das Nachtarbeitsverbot für Frauen in der Industrie, aber auch gleicher Lohn für gleiche Arbeit festgehalten. Was den Beitritt zu diesen Konventionen betrifft, so ist zumindest für die Schweiz auffallend, dass man sich tendenziell nur dort anschloss, wo die nationale Gesetzgebung schon bestand, bzw. wo die finanziellen Einbußen zu Lasten der einzelnen Arbeiterinnen gingen und keine sonstigen sozialen Kosten entstanden. Das Nachtarbeitsverbot, in der ILO Konvention 89 festgehalten, erfült diese Kriterien; die meisten europäischen Staaten traten bei.

Eine Verbesserung der Arbeits- und Lebensbedingungen von Arbeiterinnen und Arbeitern durch höhere Löhne, bessere Wohnungen, eine ausreichende ärztliche Betreuung bei Krankheit, die Verkürzung der Arbeitszeit, die Einschränkung der Nachtarbeit und die Verschärfung der Gesundheitsvorschriften in den Fabriken war notwendig. Zeitgenössische Untersuchungen begannen sich allerdings in der zweiten Hälfte des 19. Jahrhunderts zunehmend auf den Gesundheitszustand der Arbeiterinnen zu konzentrieren, der weibliche Körper wurde zum Gegenstand der Sozialpolitik. Frauen erschienen in diesem Diskurs, der hauptsächlich von Ärzten und Sozialreformern geführt wurde, als für die Industriearbeit physisch ungeeignet, und die geringere Lebenserwartung, die hohe Säuglingssterblichkeit wurden der Fabrikarbeit zugeschrieben. ${ }^{30}$ Zeitgenoss/inn/en begrüßten die gesetzliche Senkung der

$30 \mathrm{Vgl}$. Canning, Languages, wie Anm. 21, $89 \mathrm{ff}$ und Karin Hausen, Arbeiterinnenschutz, Mutterschutz und gesetzliche Krankenversicherung im Deutschen Kaiserreich und 
Arbeitsbelastung. Daneben zeigten sich aber auch schon gegen Ende des 19. Jahrhundert und vor allem im 20. Jahrhundert die Nachteile des Schutzgesetzes: Das Nachtarbeitsverbot schloss Frauen aus besser bezahlten Branchen wie z. B. der chemischen Industrie oder den Druckereien aus und wurde zudem in den Bereichen, in denen Männer und Frauen tätig waren, als Argument für niedrigere Frauenlöhne benutzt. Dass dieses gleichzeitig für Frauen die Möglichkeiten zur selbstständigen und selbstbestimmten verbesserung ihrer Situation einschränkte, wurde kaum diskutiert.

Der Anspruch der männlichen Arbeiter auf Verbesserung der Lebens- und Arbeitsbedingungen wurde mit der Notwendigkeit des männlichen „Ernährerlohns" begründet. Dieser sollte es dem Arbeiter ermöglichen, die Familie allein zu erhalten, male breadwinner zu sein, wie es in England hieß. Die Forderung zielte auf bessere Löhne. Sie war geeignet, in einer von Lohnarbeit dominierten Arbeitswelt die erwünschten Familienstrukturen zu erhalten bzw. für die Arbeiterschicht herzustellen. In einer Gesellschaft der Lohnabhängigen bedeutete der höhere Lohn die bessere gesellschaftliche Stellung sowie Dominanz und Ansehen innerhalb der Familie. Das war nur durch die Sicherung der besser bezahlten Arbeitsplätze zu erreichen, auf die der "Ernährer" Anspruch erhob. Die durch Schutzgesetze eingeschränkte, Verwendbarkeit' weiblicher Arbeitskräfte flankierte die Fortschreibung prinzipiell niedriger Frauenlöhne und machte Frauen zu ,Zuverdienerinnen“.,31

Anders als in England und Frankreich, wo die diskriminierende Wirkung der Gesetze früh und anhaltend diskutiert wurde, war in der Schweiz bis in die 20er Jahre des 20. Jahrhunderts keine Kritik zu hören. Auch in Deutschland war verhältnismäßig wenig Widerstand manifest, seitClara Zetkin 1892 ihre Haltung in dieser Frage geändert hatte und auf den Sonderschutzgesetze befürwortenden Kurs der SPD eingeschwenkt war. ${ }^{32}$ Die meisten Frauen der bürgerlichen deutschen Frauenbewegung traten für Frauenschutzgesetze ein. ${ }^{33}$ Auf den internationalen Frauenkongressen der Jahrhundertwende aber stellte die Kontroverse über Schutz oder Diskriminierung von Frauen durch spezielle Schutzbestimmungen ein wesentliches Unterscheidungsmerkmal verschiedener Richtungen der Frauenbewegung dar, ja spalteten sogar deren Kongresse. ${ }^{34}$ Radikale, mehr-

in der Weimarer Republik, in: Ute Gerhard Hg., Frauen in der Geschichte des Rechts. Von der Frühen Neuzeit bis zur Gegenwart, München 1997, 713-743, 719ff.

31 Gegen diese offensichtlichen finanziellen Einbußen als Folge von Schutzgesetzen argumentierte Margareta Gagg, wenn sie anführte, dass der allgemeine Arbeiterschutz dem Arbeiter kein „,direktes wirtschaftliches Opfer zumutet" und nur gegenüber den Arbeiterinnen „dieses Prinzip durchbrochen“ würde. Margareta Gagg, Die Frau in der Schweizerischen Industrie, Zürich/Leipzig 1928, 298. Zur Legitimation der Minderbezahlung von Frauen vgl. auch Hans Kaufmann, Die Frauenarbeit in der Schweizerischen Industrie, Zürich 1915.

32 Vgl. Karin Bauer, Clara Zetkin und die proletarische Frauenbewegung, Berlin 1978, $125 \mathrm{ff}$ und Sabine Schmitt, Der Arbeiterinnenschutz im Deutschen Kaiserreich. Zur Konstruktion der schutzbedürftigen Arbeiterin, Stuttgart/Weimar 1995, 65ff.

33 Vgl. Ulla Wikander, International Women's Congresses, 1878-1914. The Controversy over Equality and Special Labour Legislation, in: Maud L. Eduards u. a. Hg., Rethinking Change. Current Swedish Feminist Research, Uppsala 1992, 11-36, 20 , Anm. 27.

34 Vgl. Wikander, Women's Congresses, wie Anm. 33, $17 \mathrm{ff}$. 
heitlich bürgerliche Frauen argumentierten, dass die Gesetze nicht die Frauen, sondern die männlichen Privilegien schützten. ${ }^{35}$ Der Antrag der französischen Delegierten Marie Bonieval auf dem Zürcher Arbeiterschutzkongress 1897, keine speziellen Schutzgesetze für Frauen zu schaffen, war Ausdruck dieser Befürchtungen. ${ }^{36}$

IV.

Am Nachtarbeitsverbot lassen sich die Konstruktionsmechanismen, die die Geschlechterhierarchie aufrecht erhalten, ebenso verdeutlichen, wie die Wirkungsmacht der Diskurse: Die Einführung des Nachtarbeitsverbots für Frauen verstärkte die Geschlechterdifferenz auf dem Arbeitsmarkt. In der Folge unterschieden sich Männer und Frauen deutlicher auf Grund dieser gesetzlich festgelegten Verfügbarkeit. Die Geschlechterdifferenz wurde in die horizontale und vertikale Arbeitsmarktsegregation eingeschrieben. Die Segregation aber wirkt als wesentliches Element der Hierarchisierung der Arbeitsverhältnisse. Solange Männer und Frauen als Arbeitskräfte nicht austauschbar sind, ist es einfacher, die höheren Löhne und die besseren Aufstiegschancen für Männer zu legitimieren. ${ }^{37}$

Dass der tatsächliche Schutz der Frauen durch die "Schutzgesetze" eher als gering einzuschätzen ist und zudem auch immer wieder zu Gunsten „übergeordneter Werte", besonders in Kriegs- und Notzeiten aufgehoben werden konnte, legt es nahe, das Nachtarbeitsverbot in erster Linie als Diskursphänomen zu interpretieren. Als solches aber hat es die gesellschaftliche Wirklichkeit und die individuelle Arbeitssituation von Frauen nachhaltig geprägt. Dabei werden Frauen nicht - wie am Ende des 19. Jahrhunderts noch gefordert - gesetzlich von der Fabrikarbeit ausgeschlossen, aber die Gesetze verstärkten das gendering der Lohnarbeit und speziell der Fabrikarbeit. Sie bewirkten bisweilen den Ausschluss aus bestimmten Berufen und erleichterten es, Frauen auf schlechter bezahlte und insgesamt weniger attraktive Bereiche abzuschieben.

Das Nachtarbeitsverbot steht an der Schnittstelle der Definition des Normalarbeitstags, geschlechts-, gruppen- und branchenspezifischer Differenzierungen des Arbeitsmarktes, der Definition der Geschlechterrollen und der Konstruktion von Geschlecht: Das Verbot, Nachtarbeit zu verrichten, galt nur für Frauen und nur für den industriellen und gewerblichen Bereich, nicht aber für Spitäler oder private Haushalte; das Verbot fraktionierte und normierte die Arbeitszeit, ohne die ,Verwertung' männ-

35 Vgl. Wikander, Women's Congresses, wie Anm. 33, 25.

36 Vgl. Wikander, Women's Congresses, wie Anm. 33, 25. Französische Frauen hatten die diskriminierende Wirkung der Schutzgesetze zu spüren bekommen, als auf Grund des Nachtarbeitsverbots von 1892 weibliche Typografen aus den Druckereien verbannt wurden. In der Schweiz war es dann später das Verbot, mit Blei umzugehen, das den Ausschluss von Frauen aus dem gut bezahlten und gewerkschaftlich gut organisierten Druckereigewerbe zur Folge hatte. Vgl. Christine Ragaz, Die Frau in der schweizerischen Gewerkschaftsbewegung, Stuttgart 1933, 64.

37 Vgl. Karin Hausen Hg., Geschlechterhierarchie und Arbeitsteilung. Zur Geschichte ungleicher Erwerbschancen von Männern und Frauen, Göttingen 1993, Einleitung, 9f. 
licher Arbeitskraft zu beschränken. Es legitimierte Nachtarbeit von Männern; es etablierte den Normalarbeitstag und hob ihn dennoch gleichzeitig für Männer auf; es unterstützte den Anspruch von Männern auf bessere Entlöhnung in der als Nacht definierten Zeit und steigerte den Wert des männlichen Fabrikarbeiters; es gab vor, Frauen vor Doppelbelastungen zu schützen und legte sie gleichzeitig auf ihre Rolle als Hausfrauen fest; es schloss sie aus bestimmten Branchen aus und verhinderte den Erwerb von Qualifikationen; es legitimierte Lohndifferenzen und verstärkte die Arbeitsmarktsegregation.

Die Schutzgesetzgebung erfülte eine doppelte Funktion: Als Kompromiss zu einem vollständigen Verbot legitimierte sie die Fabrikarbeit für Frauen. Gleichzeitig erweiterte sie das Feld staatlicher Sozialpolitik. Sie machte Frauen zum Zentrum dieser Sozialpolitik und, zumindest in Deutschland, wohl auch zum Dreh- und Angelpunkt eines neuen Verständnisses der Aufgaben des Staates und der Rechte und Pflichten, der Zugriffs- und Kontrollmöglichkeiten staatlicher Organe.

1992 kündigten die europäischen Staaten die Konvention 89 der International Labour Organisation und machten den Weg frei zur Aufhebung des für Frauen seit dem Ende des 19. Jahrhunderts in Industriebetrieben gültigen Nachtarbeitsverbots. Damit wurde einerseits ein Kernstück der als Schutzgesetzgebung bezeichneten Arbeitsmarktregulierungen zur Disposition gestellt und der Abbau sozialpolitischer Sicherheiten eingeleitet. Andererseits fiel mit der Aufhebung des Verbots aber eine Bastion in einer Gesetzgebung, die Frauen zu einer Sonderkategorie auf dem Arbeitsmarkt werden ließ und so wesentlich die hierarchische $\mathrm{Ge}$ schlechterordnung stabilisiert hatte. Von einer Aufhebung des Verbots und der Flexibilisierung der Arbeitszeit allerdings per se eine gleichstellende Wirkung zu erwarten, erweist sich angesichts der nachhaltigen diskursiven Wirkungen des Komplexes "Frauenschutz" als unrealistisch. 